\title{
Kerstin Ruckdeschel
}

\section{Der Kinderwunsch von Kinderlosen}

Fertility intentions of childless persons

\begin{abstract}
Zusammenfassung
Mit den Daten der deutschen Population Policy Acceptance Study (PPAS) von 2003 wird der Kinderwunsch von Kinderlosen, sowie mögliche Gründe gegen eigene Kinder untersucht. Dabei stellen vor allem individuelle Werthaltungen ein entscheidendes Differenzierungskriterium zwischen Kinderlosen mit und ohne Kinderwunsch dar. Zudem bestehen zwischen den alten und den neuen Bundesländern grundsätzliche Unterschiede, sowohl was das Ausmaß und die Struktur des Kinderwunsches betrifft, als auch die Gründe gegen eine eigene Elternschaft.
\end{abstract}

Schlagworte: Kinderwunsch, Kinderlosigkeit, Werthaltungen, Gründe gegen Elternschaft

\begin{abstract}
Using data of the German Population Policy Acceptance Study (PPAS) 2003, we analyse fertility intentions of childless individuals and their motives against having a first child. We find individual value orientations as a main cause for differences between childless individuals with fertility intentions and those without. Additionally there are fundamental differences between the new and the old German Länder in what concerns the dimension and the structure of fertility intentions as well as motives against having own children.
\end{abstract}

Key words: fertility intentions, childlessness, value orientations, motives against parenthood

\section{Einleitung}

In der öffentlichen Diskussion um die Folgen des demographischen Wandels sind Kinderlosigkeit und Kinderwünsche in Deutschland derzeit nicht nur ein Thema der Fachöffentlichkeit. Dabei wird Kinderlosigkeit negativ bewertet: Kinderlose werden häufig als egoistisch dargestellt, man wirft ihnen vor, sie würden sich nur um ihr eigenes Wohlergehen kümmern und die Sorge um die nachfolgende Generation anderen überlassen, aber später von den Vorteilen der umlagefinanzierten Sozialversicherungssysteme profitieren wollen. Auch der fehlende Kinderwunsch wird thematisiert, es ist vom ,fehlenden Mut' der jüngeren Generationen die Rede oder von den ,Wenn-Dann-Müttern', die die Realisierung eines Kinderwunsches ständig auf- 
schieben. Trotz des starken Interesses ist Kinderlosigkeit aber noch relativ wenig erforscht. So konstatiert z.B. Dorbritz, ,dass die bisherige Forschung zur Kinderlosigkeit, abgesehen davon, dass wir nicht einmal ihre Ausmaße genau kennen, bei weitem nicht differenziert genug war" (Dorbritz 2003, 419). Im folgenden Beitrag sollen deshalb die Themen Kinderlosigkeit und Kinderwunsch im Mittelpunkt stehen. Nach einer kurzen Einführung zum Ausmaß der Kinderlosigkeit in Deutschland, den Gründen für und gegen eigene Kinder und zur Validität der Kinderwunschfrage werden im empirischen Teil Ergebnisse aus dem Population Policy Acceptance Survey (PPAS) vorgestellt und diskutiert. Der Fokus richtet sich auf den Kinderwunsch von Kinderlosen und deren subjektive Gründe für bzw. gegen eine eigene Elternschaft.

\section{Forschungsstand}

\section{Kinderlosigkeit in Deutschland}

Das genaue Ausmaß der Kinderlosigkeit in Deutschland kann nur geschätzt werden, da in der Bevölkerungsstatistik dazu keine Angaben vorliegen. Trotzdem gilt als sicher, dass Kinderlosigkeit in beiden Teilen Deutschlands zunimmt. Die Zahlen für Westdeutschland weisen bei Frauen der Geburtsjahrgänge 1940 bis 1965 einen Anstieg von $10 \%$ auf $28 \%^{1}$ auf, für Ostdeutschland von $9 \%$ auf $12 \%$ (Dorbritz 2005). Damit erreicht Kinderlosigkeit für die Geburtsjahrgänge nach 1965 eine Dimension, die in Europa nur noch in der Schweiz ähnlich groß ist (Dorbritz 2005). Diese Entwicklung hinterlässt auch auf der gesellschaftlichen Ebene ihre Spuren, denn die Bevölkerung spaltet sich immer mehr in einen sogenannten Familien- und einen Nicht-Familiensektor auf, mit unterschiedlichen Lebenswelten, Problemen und Bedürfnissen (Strohmeier 1993). Zum Familiensektor zählen alle privaten Lebensformen mit Kindern, d.h. sowohl Partnerschaften, unabhängig vom Institutionalisierungsgrad, als auch Alleinerziehende; zum Nicht-Familiensektor zählen entsprechend Lebensformen ohne Kinder. Der Familiensektor hat gegenwärtig den größeren Anteil an der Bevölkerung, der aber aufgrund der zunehmenden Kinderlosigkeit schrumpft (Dorbritz 2003, 406).

Die Kinderlosen, die den Nicht-Familiensektor bilden, stellen eine sehr heterogene Gruppe dar, die nach den Ursachen der Kinderlosigkeit weiter ausdifferenziert werden muss, wobei die Grenzen nicht immer klar abgrenzbar sind. Schneewind schlägt vor, bei lebenslang Kinderlosen zwischen drei Gruppen zu unterscheiden: a) bewusst Kinderlosen, b) Kinderlosen mit Kinderwunsch, der aus gesundheitlichen Gründen nicht erfüllt werden konnte und c) Kinderlosen mit Kinderwunsch, der aber solange aufgeschoben wurde, bis er aus biologischen Gründen nicht mehr erfüllt werden konnte (Schneewind 1995, 458). Dabei muss der Kinderwunsch keine Konstante im individuellen Lebenslauf sein, sondern kann sich je nach Gegebenheiten ändern. Carl weist auf das breite Kontinuum zwischen bewusster Kinderlo-

$1 \mathrm{Zu}$ unterschiedlichen Schätzergebnissen siehe z.B. Dorbritz 2005, 366 
sigkeit und lebenslang unerfülltem Kinderwunsch hin, in dem der „Kinderwunsch im Laufe des Lebens variiert und damit als Prozess zu begreifen ist" (Carl 2002, 69, vgl. auch Schneewind 1995, 463).

\section{Subjektive Gründe für und gegen eine Elternschaft}

Der Übergang zur Elternschaft hat in den letzten Jahrzehnten einen grundlegenden Wandel erfahren. Kinderhaben hat sich von einer Selbstverständlichkeit hin zu einer bewusst getroffenen Entscheidung gewandelt. Dauernd wirksame Kontrazeptiva müssen im Gegensatz zu früher bewusst abgesetzt werden, um eine Empfängnis/ Schwangerschaft zu ermöglichen. Dadurch wird die Entscheidung für ein Kind rationalisiert und gerät zu einer „Entscheidung von außergewöhnlicher Tragweite und Bedeutung“" (Rupp 2003, 79). Aus diesem Grunde stellt sich die Frage, welche Faktoren den Entschluss zur Elternschaft positiv beeinflussen. Mit der abnehmenden Selbstverständlichkeit von Kindern wird ihr subjektiv wahrgenommener Nutzen für diese Entscheidung wichtiger. In individualisierten Gesellschaften liegt er vornehmlich in der besonderen Art der Beziehung zwischen Eltern und Kindern begründet. Beck-Gernsheim (1998) spricht vom Kind als letztem „Garant(en; die Verf.) von Dauer, als Verankerung des eigenen Lebens“ (228). Auch der Value-of-ChildrenAnsatz, der den Wert von Kindern in eine ökonomische ${ }^{2}$, eine sozial-normative und eine emotionale Nutzendimension unterteilt, kommt zu ähnlichen Ergebnissen (Nauck 2001, 1989; Hoffman \& Hoffman 1973). Während den ersten beiden Dimensionen in westlichen Industriegesellschaften kaum noch Bedeutung zukommt, wird der dritte und letzte Aspekt, der emotionale Nutzen, ausschlaggebend. Kinder bieten eine emotionale Bereicherung und subjektive Sinnstiftung, die nicht durch andere Faktoren substituierbar ist. Allerdings kann der emotionale Nutzen von Kindern mit konkurrierenden Werten in Konflikt geraten (Nauck 2001, 424), weil Kinder z.B. in finanzieller und zeitlicher Hinsicht Ressourcen binden und die individuelle Mobilität und Flexibilität einschränken.

Kinder stellen gleichzeitig aber auch die letzte lebenslang bindende Verpflichtung im Leben des modernen Menschen dar. In einer bewussten Entscheidung für oder gegen Kinder werden die Chancen und Risiken einer Elternschaft für den weiteren Lebensverlauf entsprechend sorgfältig bedacht (Huinink 1995, 151). Was die Gründe gegen eine eigene Elternschaft betrifft, ist zunächst an die direkten ökonomischen Kosten zu denken. Hinzu kommen die Opportunitätskosten der Kindererziehung, die durch einen, zumeist zeitweiligen, Verzicht auf ein Gehalt oder durch Kinderbetreuungskosten entstehen. Weitere Gründe sind im Bereich Partnerschaft anzusiedeln. Allein die Existenz einer Partnerschaft begünstigt die Beschäftigung mit der Kinderwunschfrage (Huinink 2003, 10), gleichzeitig kann aber z.B. die Wahrnehmung von Elternschaft als Bedrohung der Partnerschaftsqualität auch zu einer Entscheidung gegen Kinder führen (vgl. Schneider 1999, 103). Ausschlaggebend ist, ob der Partner oder die Partnerin den Kinderwunsch teilt, was auch die realisierte Kinderzahl stark beeinflusst (Schoen et al. 1999, 798). Daneben kann der

2 Wobei dieser weiter in Arbeits- und Versicherungsnutzen unterteilt werden kann 
Wunsch nach einem egalitären Partnerschaftsmodell, meist verbunden mit einer starken Berufsorientierung der Frauen, den Übergang zur Elternschaft verhindern, da dieser aufgrund der mangelnden Möglichkeiten der Vereinbarkeit von Beruf und Familie den Wechsel zu einer stärker traditionell ausgerichteten Partnerschaft impliziert (Rupp 2005; Dornseiff \& Sackmann 2003). Schließlich können die normativen Anforderungen einer Elternschaft abschrecken, denn erhöhten Ansprüchen an die Aufgabe als Eltern - Kaufmann spricht vom „Normkomplex der verantworteten Elternschaft“ - stehen gesellschaftsbedingte „strukturelle Rücksichtslosigkeiten“ entgegen (Kaufmann 1990, 1988).

Die subjektiven Gründe gegen eine Elternschaft können sich im Lebensverlauf ändern. Eine Studie über Männer in Deutschland (Helfferich et al. 2004) zeigt, dass für jüngere kinderlose Männer mehr die Kollision mit außerfamiliären Interessen, fehlende Sicherheit oder die Angst vor zu großer Verantwortung gegen Kinder sprechen, während ältere Befragte vor allem das eigene Alter oder das der Partnerin nennen (Helfferich et al. 2004, 30). Wichtig ist bei den Gründen gegen eine Elternschaft schließlich auch die persönliche Einstellung zu Familie und Kindern. Schneewind (1995) stellt fest, dass bei bewusst Kinderlosen eine positive Einstellung zu Kindern seltener ist als bei Eltern bzw. Kinderlosen mit Kinderwunsch, während negative Erwartungen an ein Leben mit Kindern eine wesentlich wichtigere Rolle spielen.

\section{Zur Validität der Kinderwunschfrage}

Der Kinderwunsch stellt eine in die Zukunft gerichtete Handlungsabsicht dar, weshalb von Interesse ist, ob die genannte Kinderzahl tatsächlich realisiert wird. Diese Frage wurde häufig untersucht, allerdings mit widersprüchlichen Ergebnissen: So finden z.B. Noack und Østby (2002), Livi Bacci (2001) oder Coombs (1979) nur einen schwachen oder keinen Zusammenhang zwischen Wunsch und Wirklichkeit, während andere die hohe Übereinstimmung betonen (vgl. z.B. Schoen et al. 1999, Westoff 1990). Die Gründe für die unterschiedlichen Ergebnisse beginnen bereits mit der konkreten Frageformulierung. So wird die genannte Kinderzahl umso wahrscheinlicher realisiert, je enger der Zeithorizont und je konkreter die Frage ist. Daneben hängt die Realisierung eines Kinderwunsches auch von der Entwicklung der persönlichen Lebensumstände und der gesellschaftlichen Rahmenbedingungen ab. Gewollte Kinderlosigkeit wird dadurch weniger beeinflusst, was ein Grund dafür sein kann, dass sie eher verwirklicht wird als hohe Kinderwünsche (vgl. z.B. Rost 2003, 15; Rupp 2003, 85; Noack \& Østby 2002, 109).

$\mathrm{Zu}$ den Faktoren, die den Kinderwunsch beeinflussen, zählen vor allem individuelle Werthaltungen und Orientierungen (Kemkes-Grottenthaler 2004, Rost 2003, Kapella/Rille-Pfeiffer 2004), aber auch soziodemographische Merkmale wie das Alter, der Bildungsstand oder die aktuelle ökonomische Situation (Helfferich 2004,2002, Rost 2003, Kemkes-Grottenthaler 2004). Einen maßgeblichen Einfluss hat die Partnerschaft, denn für viele wird die Kinderfrage erst mit einem Partner bzw. einer Partnerin relevant, wobei der Kinderwunsch des Partners bzw. der Partnerin und die Übereinstimmung zwischen den Partnern entscheidend ist (Rupp 
2003, Schneider 1999, Thomson \& Hoem 1998, Vaskovics et al. 1996). Auch strukturelle Rahmenbedingungen wie z.B. die Möglichkeit der Vereinbarkeit von Familie und Erwerbstätigkeit (Rupp 2003, Van Peer 2002, Schoen et al. 1999, Vaskovics et al. 1996, Nave-Herz 1988) oder das soziale Umfeld (Testa \& Grilli 2006, Rupp 2005, Goldstein et al. 2004) können eine wichtige Rolle spielen. Die genannten Faktoren sind größtenteils variabel, d.h. sie können sich im individuellen Lebensverlauf ändern und entsprechend auf Ausmaß und Intensität des Kinderwunsches rückwirken.

Aufgrund der doppelten Abhängigkeit des Kinderwunsches von externen Rahmenbedingungen und individuellen Lebensumständen sowohl beim Wunsch als auch bei der Realisierung, kann der Kinderwunsch nur beschränkt als Prädiktor zukünftiger Geburtenzahlen herangezogen werden, d.h. nur unter der hypothetischen Annahme konstanter Rahmenbedingungen und stabiler individueller Einstellungen und Lebensumstände. Versteht man den Kinderwunsch aber als rein situativen Indikator, dann ermöglicht er eine Einschätzung der grundsätzlichen Bereitschaft unter den gegebenen Bedingungen Kinder zu bekommen. Der Zusammenhang von Kinderwunsch und gesellschaftlichen Rahmenbedingungen erlaubt außerdem, zumindest vorsichtige Rückschlüsse auf die Rolle einzelner Faktoren beim Übergang vom Kinderwunsch zur Elternschaft zu ziehen.

\section{Datenbasis und Methode}

\section{Daten}

Die empirischen Analysen beruhen auf der Population Policy Acceptance Study (PPAS), die von März bis Juni 2003 im Auftrag des Bundesinstituts für Bevölkerungsforschung im Rahmen des internationalen DIALOG-Projektes erhoben wurde. Inhalt der Studie ist die Frage nach der Wahrnehmung und Bewertung des demographischen Wandels und seiner Folgen in der Bevölkerung und die Zustimmung zu politischen Maßnahmen. Datenbasis ist eine repräsentative Zufallsstichprobe von 4110 Personen im Alter zwischen 20 und 65 Jahren. Die ostdeutsche Bevölkerung ist mit 2052 Personen überrepräsentiert, um für Ost-West-Vergleiche ausreichend große Fallzahlen bieten zu können. Bei getrennter Darstellung der Ergebnisse nach alten und neuen Bundesländern sind die Aussagen für das jeweilige Gebiet repräsentativ (vgl. Dorbritz et al. 2005, 59).

\section{Zur Definition des Kinderwunsches}

Die Kinderwunschfrage wurde in der PPAS nur Befragten bis zum Alter 45 gestellt und ist zweiteilig. Zuerst wurde gefragt, ob man sich überhaupt (weitere) Kinder wünscht. Die möglichen Antwortvorgaben waren ,ja“, „nein“, ,ich bin mir unsicher/ weiß nicht“" und ,ich/meine Partnerin ist schwanger“. Personen, die mit „nein“ oder „weiß nicht" geantwortet haben, wurden im Anschluss nach den Gründen ge- 
gen (weitere) Kinder gefragt, Personen mit Kinderwunsch (Schwangere inbegriffen) wurden nach der gewünschten Anzahl gefragt. Es wird davon ausgegangen, dass diejenigen, die die Frage nach einem Kinderwunsch klar bejahen, ihn im Vergleich zu Unsicheren oder ablehnenden Befragten noch am ehesten verwirklichen. Wenn es im Folgenden um den Kinderwunsch Kinderloser geht, sind deshalb nur die Personen, die mit ,ja“ geantwortet haben, gemeint. Der Kinderwunsch wird also über die Zustimmung definiert, während eine Ablehnung oder eine „Weiß nicht“Antwort als kein Kinderwunsch behandelt werden. Im gegebenen Fall wird innerhalb der Analysen auf Unterschiede zwischen Unsicheren und Kinderlosen ohne Kinderwunsch hingewiesen. In Tabelle 1 wird die Verteilung der Antworten auf die unterschiedlichen Antwortvorgaben dargestellt. Jeweils fast ein Viertel der Befragten gehört zur Gruppe der „Unsicheren“, während Schwangere nicht ins Gewicht fallen und aus den weiteren Analysen ausgeschlossen werden.

Tabelle 1: Antwortverteilung Kinderwunschfrage, Kinderlose, 20 bis 45 Jahre

\begin{tabular}{lrrrcr}
\hline \multicolumn{7}{l}{ Möchten Sie (noch weitere) Kinder, und wenn ja, wie viele? } \\
\hline \multicolumn{7}{l}{ Ja } & Nein & $\begin{array}{c}\text { Ich weiß nicht/ bin } \\
\text { mir nicht sicher }\end{array}$ & $\begin{array}{c}\text { Befragte/Partnerin } \\
\text { ist schwanger }\end{array}$ & Insgesamt \\
Westdeutschland & 266 & 215 & 147 & 7 & 635 \\
$\%$ & 42 & 34 & 23 & 1 & 100 \\
Ostdeutschland & 230 & 122 & 105 & 2 & 459 \\
$\%$ & 50 & 27 & 23 & 0 & 100 \\
\hline
\end{tabular}

Quelle: PPAS 2003; eigene Berechnungen

\section{Deskriptive Auswertungen}

Die deskriptiven Auswertungen, die vorwiegend Anteilswerte ausweisen, werden durchgehend nach Ost- und Westdeutschland getrennt durchgeführt, da die älteren Befragten ihre reproduktive Phase zumindest zum Teil noch unter den jeweils unterschiedlichen gesellschaftlichen Rahmenbedingungen durchlebt haben und sich die beiden Gebiete auch heute noch stark im reproduktiven Verhalten unterscheiden.

\section{Multivariate Auswertungen}

In der multivariaten Analyse soll die Stärke des Einflusses der differenzierenden Merkmale zwischen Kinderlosen mit und ohne Kinderwunsch überprüft werden. Der Kinderwunsch wurde dafür als binäre Variable mit den Ausprägungen ,ja, es besteht ein Kinderwunsch“ und „nein, es werden keine Kinder gewünscht; ich bin mir noch unsicher" kodiert. Dies stellt ein sehr konservatives Maß dar, so dass davon auszugehen ist, dass sich zumindest ein Teil der Unsicheren noch für Kinder entscheiden wird. Für diesen Fall einer abhängigen Variablen mit nur zwei Wertausprägungen ist die binäre logistische Regression ein bewährtes Standardverfahren (vgl. dazu z.B. Backhaus et al. 2003; Andreß et al. 1997). 
In der Ergebnistabelle (Tabelle 6) sind die relativen Odds ausgewiesen, die im konkreten Fall angeben, wie sich, ceteris paribus, die Chance verändert, zur Gruppe der Kinderlosen mit Kinderwunsch zu gehören, wenn die unabhängige Variable eine bestimmte Aussprägung hat bzw. sich um eine Einheit verändert. Ein Wert von $\operatorname{Exp}(\beta)>1$ drückt einen positiven, ein Wert von $\operatorname{Exp}(\beta)<1$ entsprechend einen negativen Zusammenhang aus, während 1 den neutralen Wert, also keinen Zusammenhang, darstellt. Mit „Chance“ sind also nicht die direkten Eintrittswahrscheinlichkeiten gemeint, sondern das Verhältnis von Eintrittswahrscheinlichkeit zur Gegenwahrscheinlichkeit.

Im Abschnitt über Gründe für und gegen eigene Kinder wurde bereits angesprochen, dass auch individuelle Werthaltungen, wie z.B. die persönliche Bedeutung von Elternschaft beim Kinderwunsch eine große Rolle spielen (vgl. auch KemkesGrottenthaler 2004; Rost 2003; Kapella \& Rille-Pfeiffer 2004, Ruckdeschel 2004). Neben dem Geschlecht, der Lebensform und der Bildung als kategorialen Variablen wurden deshalb auch individuelle Werthaltungen als metrische Merkmale in das Modell einbezogen. Aus einer Fragebatterie zur persönlichen Bedeutung von verschiedenen Lebensinhalten wurden die Faktoren „Familie und Partnerschaft“, „Wohlstand“, „Selbstverwirklichung“ und „Egalitäres Partnerschaftsideal“ gebildet (vgl. Tabelle 2).

Tabelle 2: Faktoren zur Wertedimension, alle Befragten von 20 bis 65 Jahren

\begin{tabular}{|c|c|c|c|}
\hline \multicolumn{4}{|l|}{ Faktor } \\
\hline Partnerschaft/Familie & Wohlstand & Selbstverwirklichung & $\begin{array}{l}\text { Egalitäres Partner- } \\
\text { schaftsideal }\end{array}$ \\
\hline \multicolumn{4}{|c|}{ Anteil der erklärten Gesamtvarianz } \\
\hline $15 \%$ & $13 \%$ & $13 \%$ & $12 \%$ \\
\hline \multicolumn{4}{|c|}{ Variablen: Wie wichtig ist folgendes für Sie persönlich? (Faktorladung) } \\
\hline $\begin{array}{l}\text { Seinen Kindern genug } \\
\text { Liebe und Aufmerksam- } \\
\text { keit widmen können } \\
(0,795)\end{array}$ & $\begin{array}{l}\text { In einem schönen und ge- } \\
\text { räumigen Haus leben } \\
(0,754)\end{array}$ & $\begin{array}{l}\text { Genug Zeit für seine } \\
\text { Freunde haben } \\
(0,793)\end{array}$ & $\begin{array}{l}\text { Genug Zeit für den Haus- } \\
\text { halt haben und gleichzei- } \\
\text { tig berufstätig sein } \\
(0,744)\end{array}$ \\
\hline $\begin{array}{l}\text { Seinen Kindern eine gute } \\
\text { Ausbildung ermöglichen } \\
(0,756)\end{array}$ & $\begin{array}{l}\text { Mindestens einmal im } \\
\text { Jahr Urlaub haben } \\
(0,674)\end{array}$ & $\begin{array}{l}\text { Genug Zeit für sich und } \\
\text { seine Interessen haben } \\
(0,720)\end{array}$ & $\begin{array}{l}\text { Eine gleichmäßigere } \\
\text { Aufteilung der Hausarbeit } \\
\text { von Mann und Frau } \\
(0,711)\end{array}$ \\
\hline $\begin{array}{l}\text { Mit seinem Partner in } \\
\text { Harmonie zusammenle- } \\
\text { ben } \\
(0,687)\end{array}$ & $\begin{array}{l}\text { Genügend Geld bzw. } \\
\text { Einkommen haben } \\
(0,604)\end{array}$ & $\begin{array}{l}\text { Außerhalb der eigenen } \\
\text { Familie anerkannt } u \text {. re- } \\
\text { spektiert werden } \\
(0,598)\end{array}$ & $\begin{array}{l}\text { Mann und Frau verdienen } \\
\text { jeweils ihr eigenes Geld } \\
(0,678)\end{array}$ \\
\hline $\begin{array}{l}\text { Nahestehenden Men- } \\
\text { schen Sicherheit bieten } \\
(0,658)\end{array}$ & $\begin{array}{l}\text { Im Beruf Karriere machen } \\
(0,497)\end{array}$ & $\begin{array}{l}\text { Nach Selbstverwirkli- } \\
\text { chung streben } \\
(0,416)\end{array}$ & \\
\hline
\end{tabular}

$\mathrm{KMO}^{3}$ : 780; erklärte Gesamtvarianz: 53\%; Varimax-Rotation; jeweilige Faktorladung in Klammern

Quelle: PPAS 2003; eigene Berechnungen

3 Stichprobeneignung nach Kaiser-Meyer-Olkin 


\section{Ergebnisse}

\section{Kinderlosigkeit nach Alter}

Bevor auf den Kinderwunsch von Kinderlosen näher eingegangen wird, soll zunächst die Grundgesamtheit beschrieben werden. Abbildung 1 zeigt den Anteil an Kinderlosen nach Alterskohorten getrennt für Ost- und Westdeutschland. Der Anteil Kinderloser in den neuen Bundesländern liegt dabei über die gesamte betrachtete Altersspanne hinweg unter dem in den alten Bundesländern, was nochmals das unterschiedliche Fertilitätsverhalten belegt. Zwischen 20 und 35 Jahren verlaufen die Kurven für beide Regionen relativ parallel, erst zwischen 35 und 54 vergrößert sich der Abstand. Sowohl in den alten wie auch in den neuen Bundesländern nimmt der Anteil der Kinderlosen zwischen der Altersgruppe 25 bis 29 und 30 bis 34 rapide $\mathrm{ab}$, in den neuen Bundesländern von $66 \%$ auf $40 \%$ und in den alten von $81 \%$ auf $49 \%{ }^{4}$. Es hat den Anschein, dass viele Kinderwünsche bis zum Alter von 30 Jahren verwirklicht werden. Es darf aber nicht vergessen werden, dass es sich bei der vorliegenden Studie um eine Querschnittsbetrachtung handelt. Deshalb ist davon auszugehen, dass sich in der Darstellung zwei Effekte überlagern: Einerseits der bereits beschriebene Lebenslaufeffekt, d.h. Kinderwünsche werden mit zunehmendem Alter realisiert. Andererseits wird dieser Effekt durch einen Kohorteneffekt überlagert. Durch den Geburtenaufschub der jüngeren Kohorten haben die zum Befragungszeitpunkt 30- bis 35-Jährigen ihre Kinder durchschnittlich früher bekommen als die 25- bis 29-Jährigen und haben damit bis zum Alter von 30 Jahren schon mehr Kinder (vgl. z.B. Pötzsch 2005).

Abbildung 1: Anteil der Kinderlosen nach Altersgruppen und Region

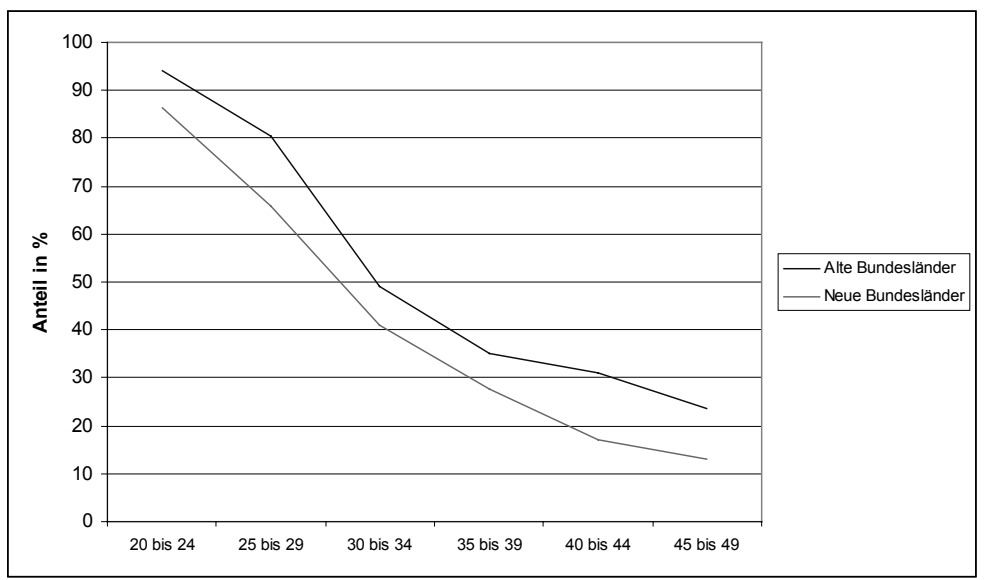

Quelle: PPAS 2003

4 Damit sind jeweils die größten Differenzen zwischen zwei Altersgruppen identifiziert 


\section{Soziodemographische Charakterisierung von Kinderlosen mit Kinderwunsch}

In Abbildung 2 ist der Anteil von Kinderlosen mit Kinderwunsch nach Alterskohorten dargestellt. Es ist zu erkennen, dass der Kinderwunsch mit steigendem Alter konstant sinkt, wobei er in den neuen Bundesländern fast durchgehend höher ist als in den alten. Wie auch beim Anteil der Kinderlosen ist wieder zwischen den Altersgruppen 25 bis 29 und 30 bis 34 der größte Rückgang zu verzeichnen. Bis zum Alter 30 oder 35 scheint ein Großteil an vorhandenen Kinderwünschen realisiert zu werden, so dass ein Selektionsprozess eintritt und in den höheren Altersstufen vermehrt kinderlose Männer und Frauen ohne oder mit aufgegebenem Kinderwunsch übrig bleiben. Interessant ist schließlich, dass in der Altersgruppe 40 bis 44 der Anteil der Befragten mit Kinderwunsch im Osten unter den im Westen fällt. Der Kinderwunsch ist in dieser speziellen Altersgruppe wegen der niedrigen Wahrscheinlichkeit einer Verwirklichung als besonders kritisch zu bewerten. Eine vertiefte Analyse müsste diese Gruppe eingehender charakterisieren und die Gründe des langen Hinausschiebens der Realisierung oder der späten Entstehung des Kinderwunsches untersuchen. Aufgrund dieser anderen Qualität des Kinderwunsches bedarf diese Gruppe einer gesonderten Betrachtung, die wegen der geringen Fallzahlen ${ }^{5}$, vor allem in der ostdeutschen Stichprobe, aber nicht möglich ist. Aus den folgenden Auswertungen wird diese Altersgruppe deshalb ausgeschlossen.

Abbildung 2: Anteil Kinderloser mit Kinderwunsch an allen Kinderlosen nach Altersgruppen und Region

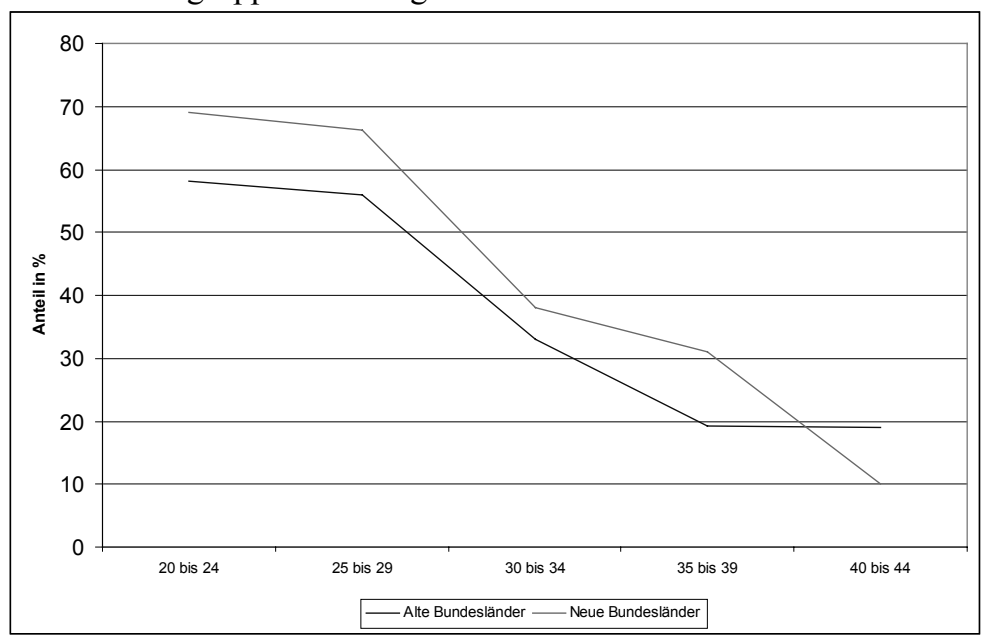

Quelle: PPAS 2003

Der Vergleich von Kinderlosen mit und ohne Kinderwunsch beschränkt sich also auf die Altersgruppen von 20 bis 39 Jahren. Diese Gruppe soll zunächst näher cha-

5 Alte Bundesländer $\mathrm{N}=15$, neue Bundesländer $\mathrm{N}=4$ 
rakterisiert werden (vgl. Tabelle 3). Da einige der betrachteten Merkmale wie Bildung oder die Lebensform typisch für bestimmte Lebensphasen sind, werden die Merkmale getrennt nach zwei Altersgruppen dargestellt.

Kinderlose in den neuen Bundesländern sind im Vergleich zu denjenigen aus den alten Bundesländern im Durchschnitt etwas jünger, was durch das niedrigere Erstgeburtsalter erklärt werden kann. Für beide Regionen gilt dagegen gleichermaßen, dass ein Selektionseffekt für das Geschlecht beobachtet werden kann. Während in den beiden jüngeren Altersgruppen die Geschlechterproportion noch nahezu ausgeglichen ist, verschiebt sie sich in den höheren jeweils deutlich zugunsten der Männer. Dies liegt zumindest teilweise daran, dass Männer ihr erstes Kind im Durchschnitt später bekommen als Frauen. Als weitere Gründe für die höhere Kinderlosigkeit von Männern werden in der Fachliteratur der Männerüberschuss in den entsprechenden Altersgruppen angeführt (Klein 2003) und die Tatsache, dass Männer sich, besonders bei nichtehelichen Kindern, seltener zur Elternschaft bekennen (Schmidt \& Winkelmann 2005, Rendall et al. 1999) oder schlicht nichts von ihrer Vaterschaft wissen (Dorbritz \& Schwarz 1996). Schließlich gilt aufgrund der immer noch bestehenden traditionellen Geschlechtsrollen, dass Männer eine Familiengründung erst bei gesicherter ökonomischer Basis erwägen (Schmitt \&Winkelmann 2005, Tölke \& Diewald 2003).

Für die alten Bundesländer bestätigt sich der bekannte Bildungseffekt, d.h. höher Gebildete sind in beiden Altersgruppen im Vergleich zur Gesamtbevölkerung häufiger kinderlos (Duschek \& Wirth 2005; Dorbritz 2003). Anders die Ergebnisse für die neuen Bundesländer, hier sind es diejenigen mit einem mittleren Bildungsniveau, die den höchsten Anteil an Kinderlosen aufweisen. Die Analyse nach Erwerbsstatus zeigt, dass neben Vollerwerbstätigkeit in den jüngeren Altersgruppen die Ausbildung eine wichtige Rolle spielt. In der höheren Altersgruppe der neuen Bundesländer ist der Anteil der Arbeitslosen bemerkenswert hoch. Was die Lebensform betrifft, zeigen sich ebenfalls deutliche Unterschiede zwischen West und Ost. Zunächst gilt für beide Regionen, dass über alle Altersgruppen hinweg Partnerlose den größten Anteil der Kinderlosen stellen, meist mit über der Hälfte der Befragten. In den alten Bundesländern sind in der jüngeren Altersgruppe Partnerschaften ohne gemeinsamen Haushalt die zweithäufigste Lebensform, in der höheren Altersgruppe verschiebt sich dieses Verhältnis dann zugunsten von nichtehelichen Lebensgemeinschaften. In den neuen Bundesländern stellen dagegen nichteheliche Lebensgemeinschaften in beiden Altersgruppen die zweitwichtigste Lebensform dar. Der Anteil an Ehen nimmt in beiden Regionen über die Altersgruppen hinweg zu, verbleibt aber auf einem relativ niedrigem Niveau, was die These der kindorientierten Eheschließung stützt, die besagt, dass, vor allem in Westdeutschland, eine nichteheliche Beziehung erst mit der Geburt eines Kindes in eine Ehe überführt wird (vgl. Nave-Herz 1984). Die unterschiedlichen Anteile von Partnerschaften ohne gemeinsamen Haushalt und nichtehelichen Lebensgemeinschaften in den alten und neuen Bundesländern verweisen auf Unterschiede, die jeweils unter den gesellschaftlichen Bedingungen der damaligen Bundesrepublik Deutschland und der ehemaligen DDR entstanden sind und weiter fortzubestehen scheinen. 
Tabelle 3: Kinderlose in West- und Ostdeutschland; Anteile in \%; 20- bis 39-Jährige

\begin{tabular}{|c|c|c|c|c|}
\hline & \multicolumn{4}{|c|}{ Kinderlose } \\
\hline & \multicolumn{2}{|c|}{ West } & \multicolumn{2}{|c|}{ Ost } \\
\hline \multicolumn{5}{|l|}{ Alter } \\
\hline$\varnothing$ & \multicolumn{2}{|c|}{28,0} & \multicolumn{2}{|c|}{27,3} \\
\hline \multirow[t]{2}{*}{ Median } & \multicolumn{2}{|c|}{27,0} & \multicolumn{2}{|c|}{25,5} \\
\hline & & & & \\
\hline Altersgruppen & $20-29$ & $30-39$ & $20-29$ & $30-39$ \\
\hline \multicolumn{5}{|l|}{ Geschlecht } \\
\hline Mann & 51 & 65 & 54 & 70 \\
\hline Frau & 49 & 35 & 46 & 30 \\
\hline \multicolumn{5}{|l|}{ Bildung $^{a}$} \\
\hline niedrig & 18 & 22 & 10 & 13 \\
\hline mittel & 33 & 33 & 47 & 53 \\
\hline hoch & 49 & 45 & 43 & 34 \\
\hline \multicolumn{5}{|l|}{ Erwerbstätigkeit } \\
\hline Vollzeit & 59 & 85 & 49 & 57 \\
\hline Teilzeit & 4 & 6 & 4 & 7 \\
\hline In Ausbildung & 26 & (2) & 29 & (6) \\
\hline Arbeitslos & 4 & 6 & 9 & 26 \\
\hline Sonstiges & 7 & (1) & 9 & $(4)$ \\
\hline \multicolumn{5}{|l|}{ Lebensform } \\
\hline Alleinlebend & 53 & 47 & 55 & 54 \\
\hline Ehe & 4 & 12 & (3) & 9 \\
\hline NEL & 16 & 21 & 23 & 24 \\
\hline LAT & 27 & 20 & 19 & 13 \\
\hline$N$ & 331 & 219 & 264 & 142 \\
\hline
\end{tabular}

a niedrig: ohne Abschluss, Hauptschulabschluss bzw. POS 8. od. 9. Klasse; mittel: Mittlere Reife bzw. POS 10. Klasse; hoch: (Fach-)Abitur

Quelle: PPAS 2003; eigene Berechnungen; ( ) Zellbesetzung $\mathrm{n}<10$

\section{Ausprägung und Umfang des Kinderwunsches von Kinderlosen}

Nach dieser kurzen Charakterisierung soll der Fokus auf dem Kinderwunsch von Kinderlosen liegen. Zunächst wird die gewünschte Kinderzahl nach Altersgruppen und alten und neuen Bundesländern getrennt dargestellt (vgl. Tabelle 4). Es fällt auf, dass bereits in der jüngsten Altersgruppe der Anteil der gewünschten Kinderlosigkeit bzw. der Unsicherheit beim Kinderwunsch sowohl in Ost- als auch in Westdeutschland relativ hoch ist; dabei ist der Anteil der Unsicheren jeweils in der Altersgruppe der 25bis 29-Jährigen am niedrigsten. Während aber in Westdeutschland diejenigen, die sicher kein Kind wollen, schon in der zweiten Altersgruppe mehr als ein Viertel ausmachen, ist dies in den neuen Bundesländern erst in der dritten Altersgruppe der Fall. Interessant sind auch die Unterschiede in der höchsten Altersgruppe: In den alten Bundesländern möchte über die Hälfte der Befragten keine Kinder mehr, in den neuen Bundesländern beträgt dieser Anteil dagegen nur gut ein Drittel, d.h. die Unsicheren sind dort wesentlich stärker vertreten. Differenzierter betrachtet zeigt sich in den alten Bundesländern eine deutliche Polarisierung beim Kinderwunsch: Entweder die Befragten möchten kein Kind bzw. sind sich unsicher oder sie wollen zwei Kinder, wobei sich die jeweiligen Anteile in den jüngeren Altersgruppen ungefähr die Waage halten, d.h. jeweils ca. $40 \%$ wollen kein Kind (oder sind sich noch unsicher) oder zwei Kin- 
der. Der Wunsch nach einer Ein-Kind-Familie ist durchgehend niedrig. Anders in den neuen Bundesländen, dort will zumindest in der jüngsten Altersgruppe eine klare Mehrheit zwei Kinder. Der Anteil der gewünschten Kinderlosigkeit (inkl. der Unsicheren) ist durchgehend niedriger als im Westen, gleichzeitig ist der Wunsch nach einer Ein-Kind-Familie über alle Altersgruppen hinweg stärker ausgeprägt. Für beide Regionen gilt, dass, wie schon in Graphik 2 sichtbar, zwischen den Altersgruppen 25 bis 29 und 30 bis 34 der größte Sprung besteht, in dem Sinne, dass ab dem Alter von 30 Jahren der Kinderwunsch ${ }^{6}$ rapide auf jeweils unter $40 \%$ sinkt. Dieser Anteil steigt in der Altersgruppe 35 bis 39 nochmals an. Die Ergebnisse erlauben für die folgenden Analysen eine Zusammenfassung der Befragten in nur noch zwei Altersgruppen.

Tabelle 4: Gewünschte Kinderzahl von Kinderlosen nach Altersgruppen, 20- bis 39-Jährige; Anteile in \%

\begin{tabular}{|c|c|c|c|c|}
\hline \multirow[b]{2}{*}{ Gewünschte Kinderzahl } & \multicolumn{4}{|c|}{ Altersgruppen } \\
\hline & 20 bis 24 & 25 bis 29 & 30 bis 34 & 35 bis 39 \\
\hline & \multicolumn{4}{|c|}{ West } \\
\hline kein Kind & 16 & 26 & 32 & 53 \\
\hline unsicher & 26 & 18 & 35 & 27 \\
\hline ein Kind & 5 & $(6)$ & $(6)$ & $(8)$ \\
\hline zwei Kinder & 40 & 41 & 24 & (2) \\
\hline drei und mehr Kinder & 13 & 9 & (3) & $(-)$ \\
\hline \multirow[t]{2}{*}{$N$} & 190 & 140 & 126 & 92 \\
\hline & \multicolumn{4}{|c|}{ Ost } \\
\hline kein Kind & 10 & 19 & 31 & 35 \\
\hline unsicher & 22 & 15 & 32 & 35 \\
\hline ein Kind & 10 & 15 & 17 & $(16)$ \\
\hline zwei Kinder & 52 & 40 & 20 & 13 \\
\hline drei und mehr Kinder & 6 & 11 & $(-)$ & (1) \\
\hline$N$ & 177 & 86 & 71 & 71 \\
\hline
\end{tabular}

Quelle: PPAS 2003; eigene Berechnungen; ( ) Zellbesetzung $\mathrm{n}<10$

Differenziert man den Kinderwunsch weiter aus (vgl. Tabelle 5), dann bestätigt sich zunächst, dass in der Altersgruppe der 20- bis 29-Jährigen über alle Ausprägungen hinweg überwiegend der Wunsch nach mindestens einem Kind vorherrscht, sich dieses Verhältnis aber in der nächsten Altersgruppe umkehrt. Unabhängig von den soziodemographischen Effekten und trotz der Querschnittsanlage der Studie deutet dies auf einen Alterseffekt hin, d.h. in der höheren Altersgruppe sind Kinderwünsche entweder erfüllt oder wurden aufgegeben, sofern sie überhaupt jemals bestanden haben. Allerdings finden sich auch hier Ausnahmen. So liegt in der jüngeren Altersgruppe der Anteil der jungen Männer mit Kinderwunsch in Westdeutschland bei 49\%, was im Vergleich zu den Männern in den neuen Bundesländern und zu den Frauen insgesamt als sehr niedrig zu bewerten ist. Dagegen haben Frauen zwischen 30 und 39 Jahren im Osten im Vergleich zu den anderen Männern und Frauen dieser Altersgruppe noch einen sehr ausgeprägten Kinderwunsch.

Betrachtet man den Kinderwunsch nach Lebensform, dann zeigt sich, dass in den alten wie den neuen Bundesländern vor allem Alleinlebende einen relativ niedrigen

6 D.h. ohne Unsichere und Befragte ohne Kinderwunsch 
Kinderwunsch haben. Wie sich auch später noch zeigen wird, ist Partnerschaft für viele eine unabdingbare Voraussetzung für Elternschaft, so dass Partnerlosigkeit eine Beschäftigung mit der Kinderfrage weitgehend verhindern kann. Nach Schmitt und Winkelmann ist ,davon auszugehen, dass Kinderwunsch und Dauer sowie Verbindlichkeit einer Partnerschaft in enger Beziehung stehen“ (Schmitt \& Winkelmann 2005, 12). Im Westen nimmt der Kinderwunsch mit steigender Institutionalisierung der Lebensform zu. In den neuen Bundesländern sind keine systematischen Unterschiede dieser Art zu finden, was auf die weiter fortgeschrittene Auflösung der institutionellen Koppelung von Ehe und Elternschaft zurückzuführen ist. Unter den 30- bis 39-Jährigen fällt im Westen der hohe Anteil von Personen in einer nichtehelichen Lebensgemeinschaft auf, der sich noch Kinder wünscht. Auch in den neuen Bundesländern liegt der Anteil der Kinderlosen mit Kinderwunsch in nichtehelichen Lebensgemeinschaften in dieser Altersgruppe vergleichsweise hoch.

Tabelle 5: Kinderwunsch von Kinderlosen nach soziodemographischen Merkmalen, 20- bis 39-Jährige; Anteil derjenigen mit Kinderwunsch in der jeweiligen Untergruppe

\begin{tabular}{|c|c|c|c|}
\hline & $\begin{array}{l}\text { Altersgruppe } \\
\text { Kinderwunsch }\end{array}$ & $\begin{array}{c}20 \text { bis } 29 \\
\%\end{array}$ & $\begin{array}{c}30 \text { bis } 39 \\
\%\end{array}$ \\
\hline & Geschlecht & & \\
\hline \multirow[t]{2}{*}{ West } & Mann & 49 & 30 \\
\hline & Frau & 67 & 23 \\
\hline \multirow[t]{3}{*}{ Ost } & Mann & 61 & 31 \\
\hline & Frau & 76 & 38 \\
\hline & Lebensform & & \\
\hline \multirow[t]{4}{*}{ West } & ohne Partner & 50 & 21 \\
\hline & Ehe & 92 & (27) \\
\hline & NEL & 67 & 48 \\
\hline & LAT & 63 & (21) \\
\hline \multirow[t]{5}{*}{ Ost } & ohne Partner & 56 & 26 \\
\hline & Ehe & (71) & $(58)$ \\
\hline & NEL & 87 & 38 \\
\hline & LAT & 80 & (39) \\
\hline & Bildung & & \\
\hline \multirow[t]{3}{*}{ West } & kein Abschluss/8.9. Klasse & 41 & (17) \\
\hline & 10. Klasse & 61 & 26 \\
\hline & (Fach-)Abitur & 59 & 35 \\
\hline \multirow[t]{4}{*}{ Ost } & kein Abschluss/8.9. Klasse & 54 & (17) \\
\hline & 10. Klasse & 62 & 33 \\
\hline & (Fach-)Abitur & 78 & 40 \\
\hline & $N$ & & \\
\hline West & & 331 & 219 \\
\hline Ost & & 264 & 142 \\
\hline
\end{tabular}

NEL: nichteheliche Lebensgemeinschaft; LAT: Living-apart-together $=$ Partnerschaft ohne gemeinsamen Haushalt

Lesebeispiel: 49\% aller Männer zwischen 20 und 29 Jahren wünschen sich mindestens ein Kind Quelle: PPAS 2003; eigene Berechnungen; ( ) Zellbesetzung $n<10$

Differenziert man den Kinderwunsch schließlich nach Bildung, dann bietet sich zunächst wieder das gewohnte Bild, d.h. Kinder werden überwiegend in der jüngeren Altersgruppe gewünscht. Zudem steigt der Kinderwunsch in allen vier betrachteten 
Teilgruppen mit zunehmender Bildung an. Hier liegt ein Selektionseffekt vor, der bei höher Gebildeten dazu führt, mit der Realisierung eines Kinderwunsches bis zum Abschluss einer Ausbildung und der Konsolidierung im Beruf zu warten (vgl. z.B. Tölke \& Diewald 2003). Allerdings sinkt mit steigendem Alter die Wahrscheinlichkeit der erfolgreichen Umsetzung eines Kinderwunsches. Obwohl also noch ca. 40\% (Ost) bzw. 35\% (West) der Befragten mit (Fach-)Abitur in der Altersgruppe 30 bis 39 Kinder wollen, ist davon auszugehen, dass sich allein aus biologischen Gründen zumindest von den Frauen nicht mehr alle diesen Wunsch erfüllen können.

\section{Einflussfaktoren auf den Kinderwunsch von Kinderlosen}

Die Stärke des Einflusses der ausgewählten Merkmale zur Unterscheidung von Kinderlosen mit und ohne Kinderwunsch wurde mit einer logistischen Regression ermittelt. Die Analyse wurde getrennt für Ost- und Westdeutschland und die beiden Altersgruppen durchgeführt (vgl. Tabelle 6). Die erklärte Varianz (Pseuo- $\mathrm{R}^{2}$ nach Nagelkerke ) zeigt, dass die ausgewählten Variablen die Unterschiede im Kinderwunsch von Kinderlosen vor allem für die jüngeren Altersgruppen und hier wiederum für die alten Bundesländer erklären.

In den deskriptiven Analysen haben sich Männer und Frauen deutlich unterschieden. Zwar bestätigt sich auch im multivariaten Verfahren, dass die Wahrscheinlichkeit eines Kinderwunsches bei jüngeren Frauen höher ist als bei Männern, während sich dieser Effekt in der höheren Altersgruppe umdreht (West) oder zumindest stark vermindert (Ost). Allerdings ist dieses Ergebnis im multivariaten Vergleich nur für die jüngeren Altersgruppen und hier auch nur noch auf dem 10\%-Niveau signifikant ${ }^{7}$ : Es konnten auch keine Wechselwirkungen mit anderen Variablen gefunden werden ${ }^{8}$.

Der Zusammenhang zwischen der aktuellen Lebensform und dem Kinderwunsch kann mit den Ergebnissen ebenfalls bestätigt werden. Dabei gilt für alle signifikanten Ergebnisse, dass partnerschaftliche Lebensformen die Wahrscheinlichkeit eines Kinderwunsches erhöhen. In der jüngeren Altersgruppe in den alten Bundesländern wird der Zusammenhang zwischen der Lebensform und dem Kinderwunsch mit zunehmender Institutionalisierung derselben stärker. Sowohl die Ehe als auch die nichteheliche Lebensgemeinschaft haben im Vergleich zu Alleinlebenden einen positiven Effekt auf den Kinderwunsch. Gerade für Ehen ist dieser Effekt in dieser Altersgruppe besonders stark, so dass der Rückschluss auf kindzentrierte EheschlieBungen nahe liegt. In der älteren westdeutschen Gruppe weist nur noch die Lebensform der nichtehelichen Lebensgemeinschaft einen signifikanten Effekt auf. In den neuen Bundesländern sind die Ergebnisse für die jüngere Altersgruppe für nichteheliche Lebensgemeinschaften und Partnerschaften mit getrennten Haushalten signifikant. Aufgrund des hohen Anteils an nichtehelichen Geburten in den neuen Bundesländern kann im Gegensatz zu den Ergebnissen für die alten Bundesländer aber nicht davon ausgegangen werden, dass eine Erfüllung des Kinderwunsches über-

7 Signifikanzniveau für West: 0,054, Ost: 0,067

8 Es wurden verschiedene Modelle berechnet, die hier aufgrund nicht signifikanter Ergebnisse nicht dargestellt werden 
durchschnittlich häufig eine weitere Institutionalisierung der Partnerschaft mit sich bringen wird. In der höheren Altersgruppe hat die Lebensform der Ehe einen positiven Effekt auf den Kinderwunsch, was aber aufgrund der geringen Zellbesetzung an dieser Stelle nicht weiter kommentiert werden soll. Wie schon in der deskriptiven Analyse dürfen die Ergebnisse nicht zu einseitigen kausalen Schlussfolgerungen verleiten. Zwar kann Partnerlosigkeit dazu führen, sich zunächst nicht weiter mit einem Kinderwunsch zu beschäftigen oder ihn aufzugeben (s.o.), ebenso ist es möglich, dass in einer Partnerschaft der Wunsch nach einer Familiengründung entsteht. Trotzdem generiert eine partnerschaftliche Lebensform per se keinen Kinderwunsch.

Schließlich bestätigt sich auch der Zusammenhang zwischen Bildungsstatus und Kinderwunsch. In der jüngeren Altersgruppe in Westdeutschland erhöht ein Abschluss nach der 10. Klasse, in der höheren Altersgruppe das (Fach-)Abitur die Wahrscheinlichkeit eines Kinderwunsches (Referenzgruppe: Personen mit niedrigerem Bildungsniveau). Ein niedrigerer Bildungsstand wird früher erreicht und führt damit auch zu einem früheren Berufseinstieg mit darauffolgender beruflicher Konsolidierung. Dies wiederum bietet bessere Rahmenbedingungen für eine Familiengründung als die eher unsichere Ausbildungsphase. Damit kann der Kinderwunsch früher umgesetzt werden. Bei Abschluss eines höheren Bildungsgrades wird der Kinderwunsch entsprechend länger als reiner Wunsch beibehalten. Im Osten Deutschlands liegt das durchschnittliche Geburtsalter immer noch etwas niedriger als im Westen (vgl. Pötzsch 2005), so dass hier bereits in der jüngeren Altersgruppe nur noch für die höchste Bildungsstufe ein signifikanter Effekt zu erkennen ist.

Entscheidend ist aber letztlich der Einfluss von individuellen Werthaltungen auf den Kinderwunsch von Kinderlosen. Die persönliche Wertschätzung von Familie und Partnerschaft hat über alle Altersgruppen und beide Regionen hinweg einen stark positiven Effekt. Von den vier untersuchten Faktoren, stellt sie die einzige Wertorientierung mit positivem Einfluss dar. Das Streben nach Selbstverwirklichung hat in der jüngeren Altersgruppe in den alten Bundesländern einen negativen Effekt, der aber im Vergleich zum positiven Einfluss des Faktors „Partnerschaft und Familie“ eher schwach ist. Schließlich wirkt sich in der jüngeren Altersgruppe im Westen auch der Wunsch nach einer egalitären Partnerschaft negativ auf den Kinderwunsch aus. Dies kann damit zusammenhängen, dass gerade in den alten Bundesländern Elternschaft für Frauen häufig einen Wechsel in eine sehr traditionell ausgeprägte Mutterrolle mit sich bringt, unabhängig von der vorherigen Rollenverteilung in der Partnerschaft (Rupp 2005; Dornseiff \& Sackmann 2003). Die Antizipation dieser Entwicklung, verbunden mit einer starken Erwerbsorientierung und einer eher geringen Wertschätzung von Familie, könnte eine völlige Abkehr von Familie und Kindern begünstigen. Diese Ergebnisse wiederholen sich für die jüngere Altersgruppe in den neuen Bundesländern, allerdings auf einem schwächeren Signifikanzniveau. Für die älteren Altersgruppen hat in beiden Teilen Deutschlands nur noch die Einstellung zu Familie und Partnerschaft einen signifikanten Einfluss. Als über alle Gruppen nicht signifikant hat sich der Faktor Wohlstand erwiesen. Es scheint also nicht so zu sein, dass Kinderlose ohne Kinderwunsch mehr Wert auf ein hohes Einkommen, eine große Wohnung, ausreichend Urlaub oder eine erfolgreiche berufliche Karriere legen und befürchten, dass Kinder dem im Wege stehen könnten. 
Zusammenfassend lässt sich an dieser Stelle festhalten, dass mit einer Ausnahme alle untersuchten Einflussfaktoren im Regressionsmodell einen signifikanten Beitrag zur Klärung der Frage leisten, ob sich Kinderlose in den entsprechenden Untergruppen ein Kind wünschen oder nicht. Ein Kinderwunsch ist bei jungen Frauen am wahrscheinlichsten. Weiter unterscheiden sich Kinderlose mit und ohne Kinderwunsch in der Lebensform, eine Partnerschaft korreliert positiv mit dem Kinderwunsch. Schließlich spielt die Einstellung zu Partnerschaft und Familie eine entscheidende Rolle. Überwiegt gerade in den jüngeren Altersgruppen das Streben nach Selbstverwirklichung, verhindert dies eher einen Kinderwunsch ebenso wie ein ausgeprägter Wunsch nach einem egalitären Partnerschaftsmodell.

Tabelle 6: Logistisches Regressionsmodell zum Kinderwunsch von Kinderlosen ${ }^{9}$

\begin{tabular}{|c|c|c|c|c|}
\hline \multirow[b]{3}{*}{ Altersgruppe } & \multicolumn{4}{|c|}{$\operatorname{Exp}\left(\beta_{j}\right)$} \\
\hline & \multicolumn{2}{|c|}{ West } & \multicolumn{2}{|c|}{ Ost } \\
\hline & $20-29$ & $30-39$ & $20-29$ & $30-39$ \\
\hline Geschlecht (Referenz: Mann) & $1,850^{+}$ & 0,689 & $1,891^{+}$ & 1,018 \\
\hline \multicolumn{5}{|c|}{ Lebensform (Referenz: ohne Partner) } \\
\hline Verheiratet & $11,112^{*}$ & 0,807 & 1,546 & $4,366^{*}$ \\
\hline NEL & $3,455^{*}$ & $3,273^{* *}$ & $3,687^{* *}$ & 2,106 \\
\hline LAT & 0,929 & 0,900 & $2,873^{*}$ & 2,151 \\
\hline \multicolumn{5}{|c|}{$\begin{array}{l}\text { Bildung } \\
\text { (Referenz: kein Abschluss/8.9. Klasse) }\end{array}$} \\
\hline 10. Klasse & $3,537^{* *}$ & 1,409 & 1,616 & 2,638 \\
\hline (Fach-)Abitur & 1,890 & $2,592^{+}$ & $3,595^{*}$ & $4,617^{+}$ \\
\hline \multicolumn{5}{|l|}{ Persönliche Bedeutung von: } \\
\hline Familie und Partnerschaft & $4,580^{\star * *}$ & $2,311^{* \star *}$ & $3,194^{* * *}$ & $2,290^{\star * *}$ \\
\hline Wohlstand & 0,779 & 1,173 & 0,960 & 1,066 \\
\hline Selbstverwirklichung & $0,360^{* * *}$ & 0,898 & $0,744^{+}$ & 1,017 \\
\hline Egalität in der Partnerschaft & $0,476^{* * *}$ & 0,852 & $0,699^{+}$ & 1,131 \\
\hline Konstante & $-0,015$ & $-1,288^{* *}$ & $-0,248$ & $-1,913^{\star *}$ \\
\hline Pseudo-R² (Nagelkerke) & 0,500 & 0,253 & 0,417 & 0,270 \\
\hline$N$ & 304 & 212 & 251 & 140 \\
\hline
\end{tabular}

sig.: ${ }^{+}<0,10 ; *<0,05 ; * *<0,01 ; * * *<0,001$

Quelle: PPAS 2003; eigene Berechnungen

\section{Gründe gegen Kinder}

In der PPAS wurden Personen ohne Kinderwunsch und diejenigen, die sich unsicher waren, schließlich auch nach ihren persönlichen Gründen gegen Kinder gefragt. Jede Antwortvorgabe konnte auf einer Skala von ,stimme sehr zu“ bis ,stimme überhaupt nicht zu“ beurteilt werden. In Tabelle 7 ist jeweils der Anteil der Befragten angegeben, die mit „stimme sehr zu“ oder „stimme zu“ geantwortet haben. Betrachtet man zunächst nur die Gründe, die allen Befragten vorgelegt wurden, dann sind es die Beibehaltung des Lebensstandards und Zukunftssorgen, die in den Augen der Befragten am stärksten gegen Kinder sprechen. Die nächstwichtigsten Gründe sind in der jüngeren

9 Da Erwerbstätigkeit keinen signifikanten Einfluss hat, wurde die Variable aus dem Modell ausgeschlossen 
westdeutschen Untergruppe die Beibehaltung des aktuellen Lebensstandards, die Beibehaltung von Freizeitinteressen und die Möglichkeit, das Leben weiterhin zu genießen. Offensichtlich stimmen sie relativ gut mit dem Faktor „Selbstverwirklichung“ aus der Regressionsanalyse überein. Anders in den drei restlichen Gruppen. Die 30- bis 39-Jährigen aus den alten Bundesländern nennen neben Zukunftssorgen die Aufrechterhaltung des aktuellen Lebensstandards und die Möglichkeit, das Leben weiterhin zu genießen. Auch bei den jüngeren Kinderlosen im Osten, die Zukunftssorgen als entscheidenden Grund gegen Kinder genannt haben, ist dies verbunden mit den hohen Kosten, die Kinder verursachen. An dritter Stelle finden sich dann auch hier die Beibehaltung des Lebensstandards und die Möglichkeit, das Leben weiterhin zu genießen. Ältere Kinderlose (zwischen 30 und 39 Jahren) in den neuen Bundesländern führen schließlich die Beibehaltung des Lebensstandards, die hohen Kosten und Zukunftssorgen an. In den neuen Bundesländern erhält die Aussage „Beibehaltung des Lebensstandards" im Lichte der weiteren häufig genannten Gründe allerdings eine völlig andere Konnotation als im Westen. Angesichts des hohen Arbeitslosenanteils in dieser Gruppe (vgl. Tabelle 3) ist davon auszugehen, dass die Aussage „Beibehaltung des Lebensstandards“ im Osten auf die Sicherung eines Mindestniveaus der Lebensführung angesichts unsicherer Zukunftsperspektiven hinweist, in der jüngeren westlichen Untergruppe dagegen eher auf die Möglichkeiten und Freiheiten eines selbstbestimmten und konsumorientierten Lebensstils.

Tabelle 7: Gründe gegen Kinder; Kinderlose ohne Kinderwunsch und Unsichere, Anteil ,stimme (sehr) zu“, 20- bis 39-Jährige

\begin{tabular}{|c|c|c|c|c|}
\hline Altersgruppe & $20-29$ & $30-39$ & $20-29$ & $30-39$ \\
\hline & & & & \\
\hline Gründe gegen Kinder: (Sehr) wichtig & & & & \\
\hline Ich möchte meinen jetzigen Lebensstandard beibehalten & 67 & 56 & 52 & 60 \\
\hline Ich mache mir zu viele Sorgen darüber, welche Zukunft meine Kinder erwartet & 51 & 61 & 67 & 56 \\
\hline Ich müsste Freizeitinteressen aufgeben & 58 & 43 & 43 & 42 \\
\hline Ein (weiteres) Kind würde zu hohe Kosten verursachen & 45 & 38 & 54 & 57 \\
\hline Ich könnte mein Leben nicht mehr so genießen wie bisher & 55 & 45 & 52 & 50 \\
\hline Ich könnte es nicht mit meiner Berufstätigkeit vereinbaren & 42 & 39 & 36 & 40 \\
\hline (Frauen) & $(48)$ & $(45)$ & $(48)$ & $(58)$ \\
\hline Mein Gesundheitszustand erlaubt es nicht & 18 & 19 & 13 & 19 \\
\hline Ich bin/Mein Partner ist zu alt & 14 & 15 & 4 & 11 \\
\hline Nur Personen ohne Partner: & & & & \\
\hline Ich lebe alleine und habe keinen festen Partner & 77 & 72 & 71. & 73 \\
\hline Nur Personen mit Partner: & & & & \\
\hline Mein Partner ist dagegen & 27 & 32 & 32 & 14 \\
\hline Meine Partnerschaft funktioniert nicht so, wie ich es mir vorstelle & 25 & 23 & 35 & 18 \\
\hline
\end{tabular}

Quelle: PPAS 2003; eigene Berechnungen

Die mangelnde Vereinbarkeit mit dem Beruf stellt in allen vier Gruppen für jeweils ca. 40\% der Befragten einen (sehr) wichtigen Grund gegen Kinder dar, betrachtet man nur Frauen, dann sind es jeweils ca. 50\%. Das Alter oder gesundheitliche Probleme werden seltener genannt. Partnerlose Befragte geben als häufigsten Grund den fehlenden Partner an, für Kinderlose in einer Partnerschaft stellt diese in einem Viertel bis einem Drittel der Fälle ebenfalls ein Hindernis für einen Kinderwunsch dar, sei es durch Probleme in der Partnerschaft oder durch Weigerung des Partners. 


\section{Diskussion}

Die Ausgangsfrage des vorliegenden Beitrags bezog sich auf den Kinderwunsch von Kinderlosen. Die Mehrheit der befragten Personen ohne Kinder möchte durchaus Kinder haben und orientiert sich am ,Normalfall' der Zwei-Kind-Familie, unabhängig ob alte oder neue Bundesländer. Ein relativ großer Anteil, in den einzelnen betrachteten Altersgruppen, jeweils im Westen mehr als im Osten, möchte aber auch kinderlos bleiben oder ist sich in dieser Frage unsicher. In Westdeutschland führt dies zu einer starken Polarisierung zwischen Personen mit und ohne Kinderwunsch, wobei diejenigen mit Kinderwunsch dann meist mindestens zwei Kinder wollen, während in Ostdeutschland auch der Wunsch nach einem Kind noch relativ stark ist.

Wie lassen sich diese beiden Gruppen mit und ohne Kinderwunsch nun voneinander abgrenzen? In der bisherigen Forschung wurde der emotionale Nutzen von Kindern als Motivation zur Familiengründung betont und auch in dieser Studie erweist sich die Familienorientierung als wichtiger diskriminierender Faktor. Die Frage, ob jemand Familie und Partnerschaft für wichtige Lebensziele hält, ist entscheidend für einen Kinderwunsch. Sobald diese Orientierung mit anderen Lebenszielen in Konflikt gerät, verringert sich die Wahrscheinlichkeit eines Kinderwunsches. Als solche weiteren Lebensziele wurden Selbstverwirklichung, Wohlstand und das Ideal einer egalitären Partnerschaft untersucht. So werden Kinder vor allem bei jüngeren westdeutschen Befragten weniger als Teil, sondern eher als Hindernis der eigenen Selbstverwirklichung wahrgenommen, was sich auch in den genannten Gründen gegen Kinder wiederfindet. Der Wunsch nach einem egalitären Partnerschaftsmodell wird ebenfalls als durch eine Familiengründung gefährdet angesehen. Ein weiterer Punkt, in dem sich Kinderlose mit und ohne Kinderwunsch unterscheiden, ist der Bildungsstand. Personen mit höherer Bildung haben häufiger einen Kinderwunsch als solche mit niedrigerer. Dies wurde mit einem Selektionseffekt erklärt: Bevor ein Kinderwunsch in die Realität umgesetzt wird, versucht man zunächst eine Ausbildung zu beenden und sich im Beruf zu konsolidieren. Da dies bei Abschluss einer höheren Bildung länger dauert, wird der Kinderwunsch entsprechend später umgesetzt oder aufgegeben. Schließlich zeigt sich im Westen auch beim Kinderwunsch die immer noch enge Koppelung von Ehe und Elternschaft.

Die bislang vorgestellten Befunde für den Einfluss von Werthaltungen auf den Kinderwunsch von Kinderlosen gelten überwiegend für die jüngeren Altersgruppen und hier wiederum für den Westen Deutschlands. Zwar gleichen sich die Einstellungen der jüngeren Altersgruppen stärker als die der älteren, trotzdem scheinen noch grundlegende Unterschiede zu bestehen, die unter anderem in den immer noch nachwirkenden unterschiedlichen ehemaligen Gesellschaftssystemen der früheren Bundesrepublik und der ehemaligen DDR begründet sind. Andererseits spiegelt sich in den Ergebnissen, besonders wenn man die angeführten Gründe gegen eine Elternschaft mit einbezieht, auch die ungünstige wirtschaftliche Situation in den neuen Bundesländern wider, die schlechte Rahmenbedingungen für die Erfüllung von Kinderwünschen bietet. Vor allem für die neuen Bundesländer, aber auch für die alten, müssen deshalb zusätzlich andere Motive untersucht werden, die stärker auf die Wahrnehmung der eigenen Zukunftschancen abzielen, was aber in diesem Beitrag aufgrund der Datenlage nicht möglich war. Schließlich lässt gerade für die neu- 
en Bundesländer eine Änderung der Rahmenbedingungen bei gleichbleibenden Werthaltungen auch eine Veränderung des Kinderwunsches erwarten. Bei den Gründen, die im Westen Deutschlands gegen Kinder genannt werden und die vor allem auf den Lebensstil betreffende, grundsätzliche Werthaltungen abzielen, scheint dies dagegen weniger wahrscheinlich.

Ich danke den anonymen Gutachtern, die mit ihren Kommentaren zur Verbesserung des Manuskriptes beigetragen haben.

\section{Literatur}

Andreß, H.-J.,Hagenaars, J.A. \& Kühnel, S. (1997). Analyse von Tabellen und kategorialen Daten. Log-lineare Modelle, latente Klassenanalyse, logistische Regression und GSKAnsatz. Berlin et al.: Springer.

Backhaus, K., Erichson B., Plinke, W. \& Weiber, R. (2003). Multivariate Analysemethoden. Eine anwendungsorientierte Einführung. Berlin u.a.: Springer (10. Auflage).

Beck-Gernsheim, E. (1998). Was kommt nach der Familie? Einblicke in neue Lebensformen. München: Beck.

Burkart, G. \& Kohli, M. (1992). Liebe, Ehe, Elternschaft. Die Zukunft der Familie. München, Zürich: Piper.

Carl, C. (2002). Kinder? Nein Danke! - Gewollt kinderlos. Reinbek bei Hamburg: Rowohlt Taschenbuch Verlag.

Coombs, L C. (1979). Reproductive goals and achieved fertility: A fifteen-year perspective. Demography 16, pp. 523-534.

Dorbritz, J. (2003). Polarisierung versus Vielfalt. Lebensformen und Kinderlosigkeit in Deutschland - eine Auswertung des Mikrozensus. Zeitschrift für Bevölkerungswissenschaft, 28, S. 403-421.

Dorbritz, J. (2005). Kinderlosigkeit in Deutschland und Europa - Daten, Trends und Einstellungen. Zeitschrift für Bevölkerungswissenschaft, 30, S. 359-408.

Dorbritz, J. \& Schwarz. K. (1996). Kinderlosigkeit in Deutschland - ein Massenphänomen? Analysen zu Erscheinungsformen und Ursachen. Zeitschrift für Bevölkerungswissenschaft, 21, S. 231-261.

Dornseiff, J.-M. \& Sackmann, R. (2003): Familien-, Erwerbs- und Fertilitätsdynamiken in Ost- und Westdeutschland. In: W. Bien, \& J. H. Marbach (Hrsg.), Partnerschaft und Familiengründung. Ergebnisse der dritten Welle des Familien-Survey. Opladen: Leske + Budrich. S. 309-348.

Duschek, K.-J. \& Wirth H. (2005). Kinderlosigkeit von Frauen im Spiegel des Mikrozensus. Wirtschaft und Statistik 8/2005, S. 800-820.

Goldstein J., Lutz, W. \& Testa, M. R. (2004). The emergence of sub-replacement family size ideals in Europe. Vienna: Vienna Institute of Demography of the Austrian Academy of Sciences (European Demographic Research Papers 2).

Helfferich, C. (2002). frauen leben. Eine Studie zu Lebensläufen und Familienplanung. Köln: Bundeszentrale für gesundheitliche Aufklärung.

Helfferich, C., Klindworth, H. \& Wunderlich, H. (2004). männer leben. Eine Studie zu Lebensläufen und Familienplanung. Köln: Bundeszentrale für gesundheitliche Aufklärung.

Hoffman, L. W. \& Hoffman, M. L., (1973). The value of children to parents. In J. T. Fawcett (Eds.), Psychological perspectives on population. New York: Basic Books, pp. 19-76.

Huinink, J. (1995). Warum noch Familie? Zur Attraktivität von Partnerschaft und Elternschaft in unserer Gesellschaft. Campus Verlag, Frankfurt/Main. 
Huinink, J. (2003). Familie und Gesellschaft. In: M. Feldhaus, N. Logemann \& M. Schlegel (Hrsg.), Blickrichtung Familie. Vielfalt eines Forschungsgegenstandes. Würzburg: Ergon Verlag. 2003. S. 1-14.

Kaufmann, F.-X. (1988). Familie und Modernität. In: K. Lüscher, F. Schultheis \& M. Wehrspaun (Hrsg.), Die „postmoderne“ Familie. Familiale Strategien und Familienpolitik einer Übergangszeit. Konstanz: UKV Verlag, 391-415.

Kaufmann, F.-X. (1990). Die Zukunft der Familie. Stabilität, Stabilitätsrisiken und Wandel der familialen Lebensformen sowie ihre gesellschaftlichen und politischen Bedingungen. München: C.H. Beck.

Kapella, O. \& Rille-Pfeiffer, C. (2004). Über den Wunsch ein Kind zu bekommen - Kinderwunsch hetero- und homosexueller Paare. Wien: Österreichisches Institut für Familienforschung (Working Paper 35).

Kemkes-Grottenthaler, A. (2004). Deteminanten des Kinderwunsches bei jungen Studierenden. Eine Pilotstudie mit explorativem Charakter. Zeitschrift für Bevölkerungswissenschaft 29, S. 193-218.

Kiefl, W. \& Schmid J. (1985). Empirische Studien zum generativen Verhalten. Erklärungsbefunde und theoretische Relevanz. Boppard am Rhein: Boldt.

Klein, T. (2003): Die Geburt von Kindern in paarbezogener Perspektive. Zeitschrift für Soziologie 32, S. 506-527.

Livi Bacci, M., (2001). Comment: Desired family size and the future course of fertility. Global fertility transition. Supplement to Population and Development Review 27, pp. 282-289.

Nauck, B. (2001). Der Wert von Kindern für ihre Eltern. „Value of Children“ als spezielle Handlungstheorie des generativen Verhaltens und von Generationenbeziehungen im interkulturellen Vergleich. Kölner Zeitschrift für Soziologie und Sozialpsychologie 53, S. 407-435.

Nauck, B. (1989). Individualistische Erklärungsansätze in der Familienforschung: die rational-choice-Basis von Familienökonomie, Ressourcen- und Austauschtheorien. In: R. Nave-Herz \& M. Markefka (Hrsg.), Handbuch der Familien- und Jugendforschung, Band 1: Familienforschung. Neuwied: Luchterhand, 45-61.

Nave-Herz, R. (1984). Familiale Veränderungen in der Bundesrepublik seit 1950. Zeitschrift für Soziologie der Erziehung und Sozialisation, 1, 45-63.

Nave-Herz, R. (Hrsg.) (1988). Kinderlose Ehen. Eine empirische Studie über die Lebenssituation kinderloser Ehepaare und die Gründe für ihre Kinderlosigkeit. München: Juventa.

Noack, T. \& Østby, L. (2002). Free to choose - but unable to stick to it? Norwegian fertility expectations and subsequent behaviour in the following 20 years. In: E. Klijzing \& M. Corijn (Eds.), Dynamics of fertility and partnership formation in Europe. Insights and lessons from comparative research. Vol. II. New York: United Nations, pp.103-116.

Pötzsch, O. (2005). Unterschiedliche Facetten der Geburtenentwicklung in Deutschland. Differenzierte Betrachtung der Geburtenstatistik als Grundlage für die Annahmen zu Bevölkerungsvorausberechnungen. Wirtschaft und Statistik 6/2005, S. 569-581.

Rendall, M. S., Clarke, L., Peters, E., Ranjit, N. \& Verropoulou, G. (1999): Incomplete reporting of men's fertility in the United States and Britain: A research note. Demography $36,135-144$.

Rost, H. (2003). Familienentwicklung und Veränderung des Kinderwunsches. In : H. Rost, M. Rupp, F. Schulz \& L. A. Vaskovics (Hrsg.), Bamberger Ehepaar-Panel. Bamberg: Staatsinstitut für Familienforschung an der Universität Bamberg (ifb-Materialien 6/2003), S. 10-20.

Ruckdeschel, K. (2004). Determinanten des Kinderwunsches in Deutschland. Zeitschrift für Bevölkerungswissenschaft 29, S. 363-386.

Rupp, M. (2003). Kinderlosigkeit. In : H. Rost, M. Rupp, F. Schulz \& L. A. Vaskovics (Hrsg.): Bamberger Ehepaar-Panel. Bamberg: Staatsinstitut für Familienforschung an der Universität Bamberg (ifb-Materialien 6/2003), S. 76-96. 
Rupp, M. (2005). Kinderlosigkeit in stabilen Ehen. Zeitschrift für Familienforschung 17, S. 2140.

Schmitt, C. (2004). Kinderlose Männer in Deutschland - Eine sozialstrukturelle Bestimmung auf Basis des Sozio-ökonomischen Panels (SOEP). Berlin: Deutsches Institut für Wirtschaftsforschung (DIW-Materialien, Band 34).

Schmitt, C. \& Winkelmann U. (2005). Wer bleibt kinderlos? Sozialstrukturelle Daten zur Kinderlosigkeit von Frauen und Männern. Berlin: Deutsches Institut für Wirtschaftsforschung (DIW-Discussion Papers 473).

Schneewind, K. (1995). Bewusste Kinderlosigkeit: Subjektive Begründungsfaktoren bei jungverheirateten Paaren. In: B. Nauck \& C. Onnen-Isemann (Hrsg), Familie im Brennpunkt von Wissenschaft und Forschung: Rosemarie Nave-Herz zum 60. Geburtstag gewidmet. Neuwied et al.: Luchterhand, S. 457-472.

Schneider, N. F. (1999). Gewollt kinderlose Ehepaare. In: Bundeszentrale für gesundheitliche Aufklärung (Hrsg.), Wissenschaftliche Grundlagen. Teil 3 - Familienplanung. Köln: Bundeszentrale für gesundheitliche Aufklärung (Band 13.3), S. 96-108.

Schoen, R., Astone, N.A., Kim, Y.J., Nathanson,C. A. \& Fields J. (1999). Do fertility intentions affect fertility behavior? Journal of Marriage and the Familiy 61, pp. 790-799.

Strohmeier, K. P. (1993). Pluralisierung und Polarisierung der Lebensformen in Deutschland. Aus Politik und Zeitgeschichte, B 17/93, S. 11-22.

Testa, R. M. \& Grilli L. (2006). L’influence des différences de fécondité dans les régions européennes sur la taille idéale de la famille. Population 61, p. 109-131.

Thomson, E. \& Hoem, J. M. (1998). Couple childbearing plans and births in Sweden. Demography 35, pp. 315-322.

Tölke, A. \& Diewald M. (2003). Berufsbiographische Unsicherheiten und der Übergang zur Elternschaft bei Männern. In: W. Bien \& J. H. Marbach (Hrsg.), Partnerschaft und Familiengründung. Analysen der dritten Welle des Familiensurveys. Opladen: Leske + Budrich, S. $349-384$

Van Peer, C. (2002). Desired and achieved fertility. In: E. Klijzing/M. Corijn (Eds.), Dynamics of fertility and partnership formation in Europe. Insights and lessons from comparative research. Vol. II. New York: United Nations, pp. 117-141.

Vaskovics, L. A., Hofmann, B., Rost, H., Schneewind, K. A., Gotzler, P., Schlehlein, B., Sierwald, W. \& Weiß J. (1996). Optionen der Lebensgestaltung junger Ehen und Kinderwunsch. (Verbundstudie - Endbericht). Bundesministerium für Familie, Senioren, Frauen und Jugend (Hrsg.), Stuttgart et al.: Kohlhammer.

Westoff, C. F., (1990). Reproductive intentions and fertility rates. International Family Planning Perspectives 16, pp. 84-89.

Eingereicht am: 22.02.2006

Akzeptiert am: 23.01.2007

\section{Anschrift der Autorin:}

Kerstin Ruckdeschel, Dipl.-Soz.

Bundesinstitut für Bevölkerungsforschung

Friedrich-Ebert-Allee 4

D-65185 Wiesbaden

E-mail: kerstin.ruckdeschel@destatis.de 